\title{
CALIDAD DE VIDA DE MUJERES EN FASE DE TRANSICIÓN MENOPÁUSICA EVALUADO POR LA MENOPAUSE RATING SCALE (MRS)
}

\author{
Fanny López A., PhD ${ }^{1 a}$, Dino Roberto Soares De Lorenzi ${ }^{2}$, Ana Cristina d'Andretta \\ Tanaka, $P h D^{36}$ \\ ${ }^{1}$ Escuela de Enfermería, Universidad Andrés Bello, Santiago, Chile. ${ }^{2}$ Departamento de Tocoginecología, Universidade \\ de Caxias do Sul, Brasil. ${ }^{3}$ Departamento de Saúde Materno-Infantil, Universidade de Sao Paulo, Brasil.
}

$\mathrm{a}_{\text {Matrona, }}{ }^{\mathrm{b}}$ Enfermera.

\section{RESUMEN}

Antecedentes: En las últimas décadas han surgido cambios en la pirámide poblacional aumentando el número de mujeres en fase climatérica, esto unido al concepto de calidad de vida, le ha dado mayor relevancia al estudio de las modificaciones que ocurren en esta etapa de la vida. Objetivos: Identificar la frecuencia e intensidad de los síntomas de la transición menopáusica. Métodos: Estudio transversal comparativo en 969 mujeres de 45 a 64 años, usuarias de consultorios de Atención Primaria de Salud del Servicio de Salud Metropolitano Occidente, Santiago, Chile. Para evaluar la severidad de los síntomas climatéricos, se utilizó la Menopause Rating Scale (MRS). Para el análisis de los datos se utilizó el test T de Student, el test no paramétrico de Chi cuadrado, el test de Mann-Whitney y un nivel de significancia de 5\%. Resultados: La edad promedio de las premenopáusicas fue de 49,1 $\pm 3,0$ años y $55,3 \pm 5,7$ años en las posmenopáusicas. El síntoma más frecuente y que se presentó con mayor intensidad fueron las molestias musculares y articulares, el dominio más afectado fue el psicológico con una intensidad moderada y el perfil socio-demográfico fue similar para ambos grupos menopáusico. Conclusión: Al considerar el puntaje global de la escala MRS, ambos grupos se clasificaron en la categoría de intensidad moderada, pero en el grupo posmenopáusico, la puntuación fue más alta, lo que se traduce en una peor calidad de vida para estas mujeres, dado principalmente por el dominio psicológico.

\section{PALABRAS CLAVES: Climaterio, menopausia, calidad de vida, escala MRS}

\section{SUMMARY}

Background: There were changes in the recent decades in the population pyramid due an increase the number of women in climacteric phase, this antecedent, connected with the concept of quality of life, has given greater relevance to the study of the changes that occur in this stage of life. Objectives: To identify the frequency and intensity of the symptoms of the menopausal transition. Methods: A cross-sectional comparative population-based study was conducted. The selection of 969 women aged 45-64 years treated in the Primary Care Health Services of the Occident Region of Santiago, Chile. The Menopause Rating Scale was used to assess the severity of climacteric symptoms. Data were analyzed using the chi-squared test, $T$ of Student test, the Mann-Whitney test; a probability of 0.05 was considered statistically significant. Results: The average age of premenopausal women were $49.1 \pm 3.0$ years and $55.3 \pm 5.7$ years in postmenopausal. 
The most frequent symptoms and of greater intensity was the muscle and joint discomfort, the domain most affected was the psychological with a moderate intensity and socio-demographic profile were similar for both menopausal groups. Conclusion: The global score of the MRS scale for both groups are classified in the category of moderate intensity, but in the postmenopausal group, the score was higher, resulting in a lower quality of life for these women, mainly due the psychological domain.

\section{KEY WORDS: Climacteric, menopause, quality of life, MRS Scale}

\section{INTRODUCCIÓN}

El proceso de transición demográfica hacia el envejecimiento de la población chilena, demanda la toma de decisiones dirigidas a la implementación de nuevas políticas públicas que atiendan la mayor expectativa de vida. El último censo del año 2002, indica un aumento de mujeres en transición menopáusica, señalando que nacionalmente, el número de mujeres entre 45 y 64 años fue de 1.428 .490 y, en la Región Metropolitana de Santiago, fue de aproximadamente 836.449 mujeres (1).

La actual preocupación por estos temas relacionados con la transición menopáusica, se originó, en gran parte, con la publicación del estudio conocido como WHI (Women's Health Initiative) en el 2002. Pese a que muchos de sus resultados han sido criticados o polemizados por problemas metodológicos y de muestreo, el WHI apunta a una asociación significativa entre el uso de terapia estroprogestativa continua y la ocurrencia de cáncer de mama y eventos cardiovasculares. Tales resultados, culminaron con la necesidad de revisar la asistencia a la mujer en el climaterio, considerando como eje principal, la calidad de vida y la individualización de las intervenciones en salud $(2,3)$.

Además, la literatura ha recalcado la importancia de tener instrumentos específicos de evaluación de la calidad de vida en el climaterio, por permitir una mayor profundidad del impacto de los síntomas menopáusicos, en esa etapa de la vida femenina $(4,5,6,7)$

Con base en la relevancia y actualidad del tema, el presente estudio, se propone identificar la frecuencia e intensidad de los síntomas de la transición menopáusica, en usuarias de los Consultorios de Atención Primaria de Salud del Servicio de Salud Metropolitano Occidente de Santiago de Chile, a través de un instrumento especifico de evaluación de la calidad de vida en el climaterio, conocido como Menopause Rating Scale (MRS) (8).

\section{PACIENTES Y MÉTODO}

Se realizó un estudio transversal, comparativo de base poblacional, con mujeres de 45 a 64 años, usuarias de los Consultorios de Atención Primaria de Salud del Servicio de Salud Metropolitano Occi- dente de Santiago de Chile, en el año 2009.

La selección de la muestra se realizó en dos fases. En la primera fase se aplicó un muestreo aleatorio simple para seleccionar los Consultorios de Atención Primaria de Salud (APS), lo que dio un total de 5 consultorios. En la segunda fase, se seleccionó el número de mujeres por consultorio, mediante un muestreo aleatorio simple, proporcional al tamaño de cobertura de la población de mujeres asignadas a cada uno de ellos. Este cálculo del tamaño de muestra, se realizo con un $98 \%$ de nivel de confianza, un $3 \%$ de error aceptable y una frecuencia del factor de estudio de $69 \%$, arrojando una muestra total de 969 mujeres (9).

En los criterios de inclusión se consideraron mujeres de 45 a 64 años con salud normal (definida por el National Center for Health Statistics, como aquella condición compatible con el desempeño de las actividades rutinarias) (10) y que cumplan con la historia menstrual solicitada. El estado de transición menopáusica fue definido según la historia menstrual de los últimos 12 meses, estableciéndose para el estudio, el estado de premenopausia, para aquellas mujeres que referían ciclos menstruales en los últimos 12 meses, y posmenopausia, para aquellas mujeres que no han tenido menstruación en el último año.

Fueron excluidas las mujeres con patologías crónicas diagnosticadas, como diabetes mellitus e hipertensión arterial, usuarias de medicamentos a base de estrógenos o progestágenos, antidepresivos y/o ansiolíticos, mujeres con patologías psiquiátricas o déficit mental que pudieran dificultar la interpretación y/o la respuesta del cuestionario. La opción por la exclusión de las usuarias de terapia hormonal u otros medicamentos a base de estrógenos o progestágenos, fue para evitar interferencias en la sintomatología climatérica o en la definición del estado menopáusico.

Los datos fueron recolectados mediante un cuestionario autoaplicado, que incluye las variables demográficas (edad, años de escolaridad y estado laboral) y la fecha de la última menstruación. Para evaluar la calidad de vida, en base a la intensidad de la sintomatología climatérica, se utilizó la Escala de Puntuación Menopáusica, Menopause Rating Scale (MRS), aplicada y validada en Chile por Aedo $y$ cols $(4,8,11)$. 
La escala está compuesta por once síntomas, agrupados en tres dominios: somático-vegetativo, psicológico y urogenital (8). El dominio somático vegetativo incluye los bochornos, molestias cardiacas, dificultades del sueño, molestias musculares y articulares. En los síntomas psicológicos, se agrupan la depresión, irritabilidad, ansiedad, agotamiento físico y mental. Por último, los síntomas urogenitales, incluye los problemas sexuales, de vejiga y sequedad vaginal.

Cada síntoma es autoevaluado por la mujer con un puntaje de 0 a 4 según el grado de intensidad: 0 = sin molestia; 1 = molestia leve; 2 = molestia moderada; 3 = molestia severa; $4=$ molestia intolerable.

Para la evaluación por dominios, se consideró el siguiente puntaje: en los dominios somático-vegetativo y psicológico: $0=\sin$ molestia; 1 a $4=$ molestia leve; 5 a $8=$ molestia moderada; 9 a $12=$ molestia severa; 13 a 16 = molestia intolerable. Para el dominio urogenital se consideró $0=$ sin molestia; 1 a $3=$ molestia leve; 4 a $6=$ molestia moderada; 7 a $9=$ molestia severa; 10 a $12=$ molestia intolerable. Para la suma total de la escala, la cual arrojaría la intensidad sintomática de cada mujer, se consideró $0=\sin$ molestia; 1 a $11=$ molestia leve; 12 a 22 = molestia moderada; 23 a 33 = molestia severa; 34 a 44 = molestia intolerable.

Las mujeres estudiadas fueron abordadas dentro del horario diurno de atención, en la sala de espera de los consultorios o en un espacio de mayor privacidad. La comprobación de las mujeres que cumplían con los criterios de inclusión, se realizaba bajo la información otorgada directamente por ellas. Posteriormente, se les explicaban los obje- tivos de la investigación y se les solicitaba la firma del consentimiento informado.

Los datos recolectados fueron analizados electrónicamente a través del programa estadístico SPSS (versión 14) y se calcularon las frecuencias simples, medias, desvío estándar y medianas.

Para el análisis de medias se utilizó el test $\mathrm{T}$ de Student. Para la comparación en proporciones, se utilizó el test no paramétrico de $x^{2}$, para la comparación de medianas, el test de Mann-Whitney. En todos los análisis realizados se aceptó un nivel de significancia de $5 \%$.

\section{RESULTADOS}

Fueron estudiadas 969 mujeres usuarias de los Consultorios de Atención Primaria de Salud de Santiago de Chile, de las cuales $18,7 \% \quad(n=181)$ eran premenopáusicas y $81,3 \%(n=788)$ posmenopáusicas.

El promedio etario se mostró naturalmente mayor en el grupo posmenopáusico $(p<0,01)$, con 55,3 $\pm 5,7$ años, mientras que, en las mujeres premenopáusicas, fue de 49,1 $\pm 3,0$ años (Tabla I). En relación a la ocupación, 49,2\% ( $n=89)$ de las entrevistadas del grupo premenopáusico y $42,9 \% \quad(n=338)$ de las posmenopáusicas, confirmaron tener una ocupación remunerada, diferencia no significativa entre los dos grupos $(p=0,12)$. En lo que se refiere a la escolaridad, se mostró similar entre las mujeres evaluadas $(p=0,34)$; el grupo posmenopáusico obtuvo en promedio $7,1 \pm 2,5$ años de estudios completados, mientras que en el grupo premenopáusico fue $7,3 \pm 2,4$ años (Tabla I).

Tabla I

CARACTERIZACIÓN DE LA POBLACIÓN ESTUDIADA

\begin{tabular}{|c|c|c|c|}
\hline Variables & Premenopausia & Posmenopausia & Valor $p$ \\
\hline \multicolumn{4}{|l|}{ Edad (en años) } \\
\hline $45-49$ & $104(57,5 \%)$ & $155(19,7 \%)$ & \multirow{4}{*}{$p<0,01^{1}$} \\
\hline $50-59$ & $66(36,5 \%)$ & $399(56,6 \%)$ & \\
\hline $60-64$ & $11(6,1 \%)$ & $234(29,7 \%)$ & \\
\hline Media \pm desvío estándar & $49,1 \pm 3,0$ & $55,3 \pm 5,7$ & \\
\hline \multicolumn{4}{|c|}{ Escolaridad (años completos de estudio) } \\
\hline Básica ( $\leq 8$ años) & $141(77,9 \%)$ & $634(80,5 \%)$ & \multirow{4}{*}{$0,34^{1}$} \\
\hline Media (9-12 años) & $36(19,9 \%)$ & $130(16,5 \%)$ & \\
\hline Superior ( $\geq 13$ años) & $4(2,2 \%)$ & $24(3,0 \%)$ & \\
\hline Media \pm desvío estándar & $7,3( \pm 2,4)$ & $7,1( \pm 25)$ & \\
\hline \multicolumn{4}{|l|}{ Trabajo remunerado } \\
\hline $\operatorname{Sin}$ & $89(49,2 \%)$ & $338(42,9 \%)$ & \multirow[t]{2}{*}{$0,12^{2}$} \\
\hline Con & $92(50,8 \%)$ & $450(57,1 \%)$ & \\
\hline Total & 181 & 788 & \\
\hline
\end{tabular}

${ }^{1}$ Test de T Student . ${ }^{2}$ Chi cuadrado 
La frecuencia de la sintomatología climatérica se presentó elevada, sin grandes diferencias entre los dos grupos evaluados, afectando en un $99,4 \%$ al grupo premenopáusico y en $98,9 \%$ al posmenopáusico (Tabla II). Entre los síntomas más frecuentes destacan las molestias musculares y articulares (indicadas por un $80,1 \%$ de las mujeres premenopáusicas y un $85,3 \%$ de las posmenopáusicas), seguidos del cansancio físico y mental (76,2\% y $81,5 \%$, respectivamente). Entre los síntomas menos referidos se encuentra la sequedad vaginal $(44,8 \%$ de las premenopáusicas y $54,6 \%$ de las posmenopáusicas) y los problemas sexuales $(50,8 \%$ de las premenopáusicas y $58,1 \%$ de las posmenopáusicas).

La tendencia a la mayor severidad de la sintomatología climatérica en los años que siguen a la menopausia puede ser vista en la Figura 1, en la cual, el grupo premenopáusico, tiene una sintomatología que va de ausente a leve en un $48,1 \%$ y moderada a severa en un $50,8 \%$ de esas mujeres.

Tabla II DISTRIBUCIÓN DE LA POBLACIÓN ESTUDIADA EN RELACIÓN A LA FRECUENCIA DE LOS
SÍNTOMAS REFERIDOS SEGÚN ESTADO MENOPÁUSICO

\begin{tabular}{|c|c|c|c|c|c|c|}
\hline Síntomas & $\operatorname{Sin}$ & Leve & Moderado & Severo & Intolerable & $\mathrm{p}^{*}$ \\
\hline \multicolumn{7}{|l|}{ Bochornos: } \\
\hline Premenopausia & $43,1 \%$ & $29,8 \%$ & $15,5 \%$ & $7,7 \%$ & $3,9 \%$ & \multirow[t]{2}{*}{0,27} \\
\hline Postmenopausia & $34,9 \%$ & $32,0 \%$ & $18,8 \%$ & $10,8 \%$ & $3,6 \%$ & \\
\hline \multicolumn{7}{|l|}{ Molestias cardiacas: } \\
\hline Premenopausia & $46,4 \%$ & $31,5 \%$ & $17,1 \%$ & $4,4 \%$ & $0,6 \%$ & \multirow[t]{2}{*}{0,08} \\
\hline Posmenopausia & $36,2 \%$ & $32,9 \%$ & $23,1 \%$ & $7,1 \%$ & $0,8 \%$ & \\
\hline \multicolumn{7}{|c|}{ Dificultades del sueño: } \\
\hline Premenopausia & $33,1 \%$ & $30,9 \%$ & $19,9 \%$ & $13,8 \%$ & $2,2 \%$ & \multirow[t]{2}{*}{0,50} \\
\hline Posmenopausia & $27,7 \%$ & $30,2 \%$ & $25,1 \%$ & $14,7 \%$ & $2,3 \%$ & \\
\hline \multicolumn{7}{|c|}{ Molestias musculares y articulares: } \\
\hline Premenopausia & $19,9 \%$ & $24,3 \%$ & $24,9 \%$ & $23,2 \%$ & $7,7 \%$ & \multirow[t]{2}{*}{0,40} \\
\hline Posmenopausia & $14,7 \%$ & $25,3 \%$ & $28,3 \%$ & $25,5 \%$ & $6,2 \%$ & \\
\hline \multicolumn{7}{|l|}{ Depresión: } \\
\hline Premenopausia & $30,9 \%$ & $27,1 \%$ & $25,4 \%$ & $13,3 \%$ & $3,3 \%$ & \multirow[t]{2}{*}{0,54} \\
\hline Posmenopausia & $26,1 \%$ & $26,4 \%$ & $30,8 \%$ & $14,1 \%$ & $2,5 \%$ & \\
\hline \multicolumn{7}{|l|}{ Irritabilidad: } \\
\hline Premenopausia & $23,2 \%$ & $35,9 \%$ & $26,0 \%$ & $10,5 \%$ & $4,4 \%$ & \multirow[t]{2}{*}{0,15} \\
\hline Postmenopausia & $25,9 \%$ & $27,8 \%$ & $27,8 \%$ & $15,2 \%$ & $3,3 \%$ & \\
\hline \multicolumn{7}{|l|}{ Ansiedad: } \\
\hline Premenopausia & $27,6 \%$ & $33,7 \%$ & $22,7 \%$ & $12,7 \%$ & $3,3 \%$ & \multirow[t]{2}{*}{0,31} \\
\hline Postmenopausia & $24,7 \%$ & $29,3 \%$ & $28,2 \%$ & $15,6 \%$ & $2,2 \%$ & \\
\hline \multicolumn{7}{|c|}{ Cansancio físico y mental: } \\
\hline Premenopausia & $23,8 \%$ & $35,9 \%$ & $23,2 \%$ & $14,4 \%$ & $2,8 \%$ & \multirow[t]{2}{*}{0,12} \\
\hline Posmenopausia & $18,5 \%$ & $31,2 \%$ & $31,1 \%$ & $17,1 \%$ & $2,0 \%$ & \\
\hline \multicolumn{7}{|l|}{ Problemas sexuales } \\
\hline Premenopausia & $49,2 \%$ & $21,0 \%$ & $14,4 \%$ & $11,6 \%$ & $3,9 \%$ & \multirow[t]{2}{*}{0,43} \\
\hline Posmenopausia & $41,9 \%$ & $20,3 \%$ & $19,5 \%$ & $16,6 \%$ & $1,6 \%$ & \\
\hline \multicolumn{7}{|l|}{ Problemas de vejiga: } \\
\hline Premenopausia & $45,9 \%$ & $29,8 \%$ & $13,3 \%$ & $9,4 \%$ & $1,7 \%$ & \multirow[t]{2}{*}{0,19} \\
\hline Posmenopausia & $39,3 \%$ & $30,6 \%$ & $20,6 \%$ & $7,7 \%$ & $1,8 \%$ & \\
\hline \multicolumn{7}{|l|}{ Sequedad vaginal: } \\
\hline Premenopausia & $55,2 \%$ & $27,1 \%$ & $9,4 \%$ & $6,6 \%$ & $1,7 \%$ & \multirow[t]{2}{*}{0,11} \\
\hline Posmenopausia & $45,4 \%$ & $33,4 \%$ & $14,1 \%$ & $5,8 \%$ & $1,3 \%$ & \\
\hline
\end{tabular}

*Test de chi cuadrado 


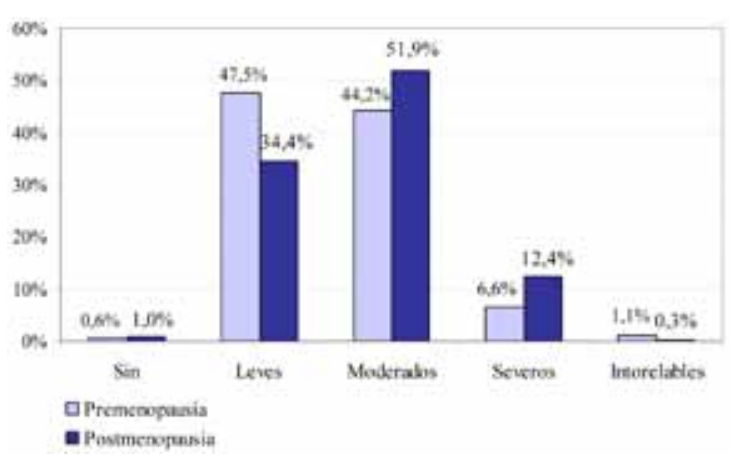

Figura 1. Distribución de las mujeres según la severidad de los síntomas climatéricos.

Mientras que entre las mujeres postmenopáusicas, los síntomas climatéricos se mostraron de ausentes a leves en un $35,4 \%$ y, con un claro aumento, de moderados a severos en un $64,3 \%$ de los casos.

En relación a la frecuencia de las quejas climatéricas, según los dominios de síntomas que componen la escala MRS, se verificó que los más frecuentes fueron los de naturaleza psicológica, siendo mencionados por un $98,3 \%$ de las entrevistadas premenopáusicas y un $95,3 \%$ de las posmenopáusicas. En tanto, los síntomas relacionados con el carácter somato-vegetativo fueron apuntados por $94,5 \%$ de las mujeres premenopáusicas y $95,2 \%$ de las posmenopáusicas. Las quejas relacionadas a la esfera urogenital, fueron indicadas por un $79 \%$ del grupo premenopáusico y un $83,2 \%$ del posmenopáusico.

En ambos grupos, se constató que existe un deterioro moderado en la calidad de vida, relacionado principalmente con los síntomas de naturaleza psicológica, alcanzando puntajes medianos de 5,4 en las mujeres premenopáusicas y de 5,8 en las posmenopáusicas. En relación al dominio somático, la mediana en el grupo premenopáusico fue de 4,8 (intensidad leve) y de 5,3 en el grupo postmenopáusico (intensidad moderada). En lo que corresponde a los síntomas urogenitales, estos se mostraron de intensidad leve (mediana $=2,6$ ) entre las mujeres premenopáusicas y moderados en el grupo posmenopáusico (mediana=3,0) (Tabla III).

Finalmente, al analizar comparativamente los índices globales de calidad de vida, estos revelaron puntajes significativamente peores en el grupo posmenopáusico en todos los dominios que componen la escala MRS (Tabla III).

\section{DISCUSIÓN}

La preocupación por lo relacionado con la calidad de vida es un fenómeno reciente, y en gran parte como consecuencia del aumento de la expectativa de vida observado en las últimas décadas. La definición de calidad de vida, particularmente en el ámbito de la salud, es todavía controversial. Para la Organización Mundial de la Salud, este término se relaciona con la manera en que los individuos perciben su propia vida dentro de un contexto cultural, según sus expectativas, deseos e intereses personales. Dentro de las dimensiones que le dan significado, se destacan la mantención de la capacidad funcional, la satisfacción general y el estado emocional, aspectos particularmente significantes en el periodo del climaterio (12).

En el presente estudio, los puntajes de calidad vida, en todos los dominios que componen el instrumento de evaluación MRS (esfera somática, emocional e urogenital/sexual), se muestran significativamente más deteriorados en el grupo posmenopáusico. Destaca también la constatación de la

Tabla III

CALIDAD DE VIDA POR DOMINIO (MRS), SEGÚN ESTADO MENOPÁUSICO

\begin{tabular}{llcccccc}
\hline $\begin{array}{c}\text { Dominios } \\
\text { (MRS) }\end{array}$ & $\begin{array}{c}\text { Estado } \\
\text { menopáusico }\end{array}$ & Promedio & $\begin{array}{c}\text { Desvío } \\
\text { estándar }\end{array}$ & Mediana & \multicolumn{2}{c}{$\begin{array}{c}\text { Porcentaje } \\
25 \%\end{array}$} & $\mathrm{p}^{*}$ \\
\hline Somático & Premenopausia & 4,8 & 3,2 & 4,0 & 2,0 & 6,0 & $<0,01$ \\
& Posmenopausia & 5,3 & 3,1 & 5,0 & 3,0 & 7,0 & \\
Psicológico & Premenopausia & 5,4 & 3,4 & 5,0 & 3,0 & 7,0 & $<0,05$ \\
& Posmenopausia & 5,8 & 3,3 & 6,0 & 3,0 & 8,0 & \\
Urogenital & Premenopausia & 2,6 & 2,4 & 2,0 & 1,0 & 4,0 & $<0,01$ \\
& Posmenopausia & 3,0 & 2,2 & 3,0 & 1,0 & 5,0 & \\
Resultado total & Premenopausia & 12,8 & 12,8 & 12,0 & 7,0 & 18,0 & $<0,01$ \\
& Posmenopausia & 14,2 & 6,9 & 14,0 & 9,0 & 19,0 & \\
\hline
\end{tabular}

* Test de Mann-Whitney 
preocupante frecuencia de síntomas climatéricos que aquejan a las mujeres entrevistadas, con mayor severidad al grupo posmenopáusico, lo cual es superior al $98 \%$. Los datos arrojados se asemejan al porcentaje de la mayoría de los países de la cultura occidental (13-17). Pese a la importancia del tema, al revisar la literatura encontramos que los estudios publicados, utilizando la escala MRS, se iniciaron recientemente en la actual década, como es en un trabajo realizado a nivel continental, en los años 2001-2002, el cual mostró puntajes más bajos que lo observado en nuestro estudio: 7,6 en Asia y 8,8 en Europa. En los Estados Unidos este valor fue de 9,1 y en América Latina fue de 10,4 (5), es decir la calidad de vida se encontraba menos afectada, que en las mujeres de nuestro estudio (12,8 en premenopáusicas y 14,2 en posmenopáusicas).

Posteriormente, en un estudio multicéntrico (2006-2007) que evaluó a 8.373 mujeres saludables con edades entre 40 a 59 años residentes en 18 ciudades de 12 países latinoamericanos a través del instrumento MRS, reveló que los peores puntajes con base en la sintomatología climatérica fueron observados en Chile y Uruguay con porcentajes de síntomas moderados a severos de $80,8 \%$ y $67,4 \%$ respectivamente (14), lo que al comparar con nuestro estudio, las mujeres posmenopausicas presentaron aproximadamente $64 \%$, síntomas que van de moderado a severo, lo que es superior a los valores estándar mundiales informados (18), hallazgo preocupante. Además, de lo anterior, se afirma que las mujeres latinoamericanas tienen una edad de menopausia más precoz que las mujeres europeas y norteamericanas, trayendo por consecuencia que nuestras mujeres se expongan más tiempo a la sintomatología derivada del efecto del hipoestrogenismo (19).

Es evidente, con la literatura estudiada y con los datos encontrados por el presente estudio, que la mujer chilena es una de las más afectadas en su calidad de vida, observando que el síntoma más frecuente en la presente casuística, fue el de las molestias musculares y articulares, tanto para las mujeres premenopáusicas como para las posmenopáusicas, además de ser el de mayor severidad en ambas fases de la menopausia. El mismo resultado se dio en Sri Lanka, en un estudio con 683 mujeres de 45 a 60 años (2009), donde esta molestia estuvo presente en un $62,5 \%$ y un $76,7 \%$ de las pre y posmenopáusicas, respectivamente (20). De igual manera, un estudio realizado con 1.189 mujeres de Nigeria (2009), con edad entre 40 y 60 años, arrojó la prevalencia de este síntoma presente en un $51,7 \%$ de las premenopáusicas y en un $65,1 \%$ de las postmenopáusicas (21). Merece atención que este síntoma, la literatura lo asocia a los cambios físicos que acompañan el envejecimiento, los estilos de vida, y el estado general de salud de la mujer, lo que se confunde con el estado menopausal (22).

En relación a los dominios medidos en nuestro estudio por esta escala MRS, se detecta un mayor deterioro del dominio psicológico en ambas fases con compromiso principalmente del estado depresivo y con tendencia al agravamiento después de la menopausia, lo que según De Lorenzi y cols (23), puede ser como resultado de la mayor labilidad emocional, derivado de una declinación en los niveles séricos de estradiol que llevan a una reducción en la secreción de endorfinas cerebrales. En Chile, se tiene como antecedente un estudio realizado en 370 mujeres por Prado y cols (15), cuyos puntajes se muestran próximos a los nuestros, pero mas comprometidos en relación a la severidad de los síntomas de las mujeres postmenopáusicas. De manera similar en Brasil, De Lorenzi y cols (24), realizaron un estudio a 133 mujeres premenopáusicas y 103 posmenopáusicas, los resultados globales no mostraron grandes diferencias entre los grupos estudiados $(p=0,12)$, sin embargo, en el grupo de mujeres premenopáusicas hacían mayor referencia a los síntomas psicológicos $(p=0,05)$, mientras que las mujeres posmenopáusicas evidenciaban mayor sintomatología somato- vegetativa $(p<0,01)$ y urogenitales $(p=0,01)$.

Contrastando estos resultados, existe un estudio realizado en Campinas, Brasil, por Pedro y cols (25), en el cual sus resultados incluyen una elevada prevalencia de síntomas psicológicos, cuya intensidad no se correlacionaba con el estado menopáusico de las mujeres investigadas.

La hipótesis más admisible seria que la severidad de los síntomas climatéricos se relaciona de la interacción del nivel de hipoestrogenismo con factores culturales y psicológicos, así referidos por De Lorenzi y cols (26), quienes evaluaron la sintomatología de 254 mujeres posmenopáusicas usuarias del Consultorio de Climaterio de la Universidad de Caxias do Sul, Brasil. Su análisis multivariado reveló que las mujeres con historia de actividad física regular, caucásicas y con una percepción o actitud positiva frente a la menopausia, manifestaban una sintomatología climatérica significativamente menos severa, reforzando la influencia de factores psicosociales y culturales.

Al caracterizar el perfil sociodemográfico de las mujeres de nuestro estudio, se tiene que los antecedentes de ocupación y años de escolaridad son similares. Estos resultados se condicen con la realidad de la ciudad en que viven, Santiago, que de acuerdo al último censo (2002) cuenta con un 
$69,1 \%$ de mujeres de 40 a 49 años y un $47,4 \%$ de mujeres de 50 y más años que han cursado como mínimo la enseñanza básica completa. Por otra parte, en lo que respecta a su ocupación, casi la mitad de nuestra población de estudio, trabaja con remuneración, situación similar al $43,3 \%$ de las mujeres de 45 a 54 años que viven en Santiago (1). Estos datos indican poca variación entre las mujeres pre y posmenopáusicas, lo que evidencia que estos factores por sí solos no harían la diferencia entre ambos grupos en torno a la sintomatología climatérica.

\section{CONCLUSIÓN}

El perfil etario de nuestras mujeres es del orden de los 49 años y menos en las mujeres premenopáusicas y de 50 años y más, en las posmenopáusicas. Para ambos grupos, el síntoma más frecuente y que se presenta con mayor intensidad, es el de las molestias musculares y articulares. En relación a los dominios, el más afectado es el psicológico con una intensidad moderada. Al considerar el puntaje global de la escala MRS, ambos grupos se clasifican en la categoría de intensidad moderada, pero en el grupo posmenopáusico, la puntuación es más alta, lo que se traduce en una peor calidad de vida para estas mujeres, dado principalmente por el dominio psicológico.

\section{BIBLIOGRAFÍA}

1. Departamento de Estadísticas e Información de Salud - DEIS. Ministerio de Salud de Chile. Población Censo 2002.

2. McGowan JA, Pottern L. Commentary on the Women's Health Initiative. Maturitas 2000;34:109-12.

3. Nabel EG. The women's health initiative. Science 2006;313:1703.

4. Heinemann L, Potthoff P, Schneider HP. International versions of the Menopause Rating Scale (MRS). Health Qual Life Outcomes 2003;1:28-34.

5. Heinemann K, Ruebig A, Potthoff $P$, Schneider $H$, Strelow F, Heinemann L, et al. The Menopause Rating Scale (MRS): A methodological review. Health Qual Life Outcomes 2004;2:45-52.

6. Heinemann L, Dominh T, Strelow F, Gerbsch S, Schnitker J, Schneider HPG. The Menopause Rating Scale (MRS) as outcome measure for hormone treatment? A validation study. Health Qual Life Outcomes 2004;2:67-73.

7. Dinger J, Zimmermann T, Heinemann L, Stoehr D. Quality of life and hormone use: new validation results of MRS scale. Health Qual Life Outcomes 2006;2:32-6.

8. Berlin Center for Epidemiology and Health Research.
Evaluation \& reference values. MRS - the menopause rating scale 2008. Disponible en: www.menopauserating-scale.info/evaluation.htm (Consultado el 29 de abril de 2009).

9. Freedman R. Hot Flush Etiology: New Directions for Research. Menopause 2002;11:36-41.

10. Brett KM, Chong Y. Hormone replacement therapy: knowledge and use in the United States. Hyattsville, Maryland: National Center for Health Statistic. 2001.

11. Aedo S, Porcille A, Irribarra C. Calidad de vida relacionada con el climaterio en una población chilena de mujeres saludables. Rev Chil Obstet Ginecol 2006;71: 402-9.

12. Seide EMF, Zannon CMLC. Qualidade de vida e saúde: aspectos conceituais e metodológicos. Cad Saúde Pública 2004;20:580-8.

13. Chedraui P, Aguirre W, Hidalgo L, Fayad L. Assessing menopausal symptoms among healthy middle aged women with the Menopause Rating Scale. Maturitas 2007;57:271-8.

14. Chedraui $P$, Blümel JE, Baron G, Belzares E, Bencosme A, Calle A, et al. Impaired quality of life among middle aged women: a multicentre Latin American study. Maturitas 2008;61:323-9.

15. Del Prado M, Fuenzalida A, Jara D, Figueroa R, Flores $\mathrm{D}$, Blümel JE. Evaluación de la calidad de vida en mujeres de 40 a 59 años mediante la escala MRS (Menopause Rating Scale). Rev Méd Chile 2008;136:1511-7.

16. Polisseni A, Teixeira S, Grünewald T, Fernandes E, De Castro L. Perfil das Participantes do Projeto de Extensão "Viver Melhor - Assistência Integral às Mulheres no Climatério. HU Revista, Juiz de Fora 2009;35:19-24.

17. Kakkar V, Kaur D, Chopra K, Kaur A, Kaur IP. Assessment of the variation in menopausal symptoms with age, education and working/non-working status in north-Indian sub population using menopause rating scale (MRS). Maturitas 2007;57:306-14.

18. Berlin Center for Epidemiology and Health Research. Evaluation \& reference values. MRS - the menopause rating scale 2008. Disponible en: www.menopause-rating-scale.info/documents/Ref_Values_CountrGr.pdf . (Consultado el 29 de enero de 2009)

19. Castelo-Branco $C$, Blümel JE, Chedraui $P$, Calle A, Bocanera R, Depiano E, et al. Age at menopause in Latin America. Menopause 2006;13:706-12.

20. Waidyasekera H, Wijewardena K, Lindmark G, Naessen $\mathrm{T}$. Menopausal symptoms and quality of life during the menopausal transition in Sri Lankan women. Menopause 2009;16:164-70.

21. Olaolorun FM, Lawoyin TO. Experience of menopausal symptoms by women in an urban community in Ibadan, Nigeria. Menopause 2009;16:822-30.

22. Chim H, Tan BHI, Ang CC, Chew EMD, Chong YS, Saw $\mathrm{SM}$. The prevalence of menopausal symptoms in a community in Singapore. Maturitas 2002;41(4):275-82. 
23. De Lorenzi DRS, Catan LB, Moreira K, Ártico GR. Assistência à enferm 2009;62(2):287-93.

24. De Lorenzi DRS, Binelli L, Cusin T, Felini R, Bassani $F$, Arpini AC. Caracterização da qualidade de vida segundo o estado menopausal entre mulheres da Região Sul do Brasil. Rev Bras Saúde Matern Infant Recife 2009;9:459-66.
25. Pedro AO, Pinto-Neto AM, Costa-Paiva L, Osis MJ, Hardy E. Procura de serviiço médico por mulheres climatéricas brasileiras. Rev Saúde Pública 2003; 36:484-90.

26. De Lorenzi DRS, Danelon C, Saciloto B, Padilha Jr I. Fatores indicadores da sintomatologia climatérica. Rev Bras Ginecol Obstet 2005;27:12-9. 